\section{Manejo quirúrgico de la transmigración bilateral de caninos mandibulares.}

\section{Reporte de caso}

\section{Surgical management of bilateral transmigration of mandibular canines.}

\section{Case report}

\section{Caso Clínico}

Laurent Coello de la Cruz ${ }^{1, a}$, Fernando Fernandez Cáliz ${ }^{1, b}$ Natalia Martínez Rodríguez 1,c, Javier Sanz Alonso 1,b, José Martínez-González ${ }^{1, d}$, Cristina Barona Dorado 1,

${ }^{1}$ Universidad Complutense de Madrid, Facultad de Odontología, Departamento de Especialidades Clínicas Odontológicas. Madrid, España.

a Cirujana Dentista.

${ }^{\mathrm{b}}$ Doctor en Odontología.

c Doctora en Odontología.

d Doctor en Medicina y Cirugía, Doctor en Odontología y Doctor en Veterinaria.

\section{Correspondencia:}

Laurent Coello de la Cruz

Correo electrónico: I.coello@hotmail.com

C/Riobamba No 8 Bajo C, 28027 Madrid. España.

\section{Coautores:}

Fernando Fernandez Cáliz

fernanfe@ucm.es

Natalia Martínez Rodríguez

nataliamartinez@ucm.es

Javier Sanz Alonso

jsanzalonso@ucm.es

José Martínez-González

jmargo@ucm.es

Cristina Barona Dorado

cbarona@ucm.es

\section{Editora:}

Dahiana Alfaro Carballido

Universidad de San Martín de Porres, Perú.

nado, traspasa en más de la mitad de su longitud la línea media. Es una anomalia frecuente y es aún más raro que ocurra de forma bilateral. Se presenta con mayor incidencia en los caninos mandibulares, en el sexo femenino, de forma unilateral, y siendo el canino izquierdo el más comúnmente afectado. El objetivo de este trabajo es describir el manejo quirúrgico satisfactorio en un paciente varón de 16 años, asintomático, el cual presentaba a la exploración clínica intraoral ausencia de ambos caninos mandibulares permanentes y persistencia de canino temporal inferior izquierdo, acompañado de una ligera malposición dentaria. En la exploración radiográfica (panorámica), se observaron ambos caninos inferiores derecho e izquierdo, en posición horizontal, bajo los ápices de incisivos inferiores. Se procedió a la extracción quirúrgica de ambos caninos, transcurriendo el postoperatorio sin incidencias. Se realizaron controles periódicos y radiográficos satisfactorios a los seis meses.

Conflicto de intereses: los autores declaran no tener conflictos de interés.

Fuente de financiamiento: autofinanciado

Recibido: 28/01/19

Aceptado: $14 / 03 / 19$

Publicado: 05/06/19

Palabras clave: Diente impactado; Migración del diente; Diente incluido; Canino (fuente: DeCS BIREME).

\begin{abstract}
Transmigration is defined as the phenomenon in which an unerupted included tooth transcends the midline by more than half of its length. It is a rare anomaly and it is even more rare to occur bilaterally. It is presented with greater incidence in the mandibular canines, in the female sex, unilaterally, and the left canine being the most commonly affected. The objective of this study is to describe satisfactory surgical management in a 16-year-old asymptomatic male patient, which presented to the intraoral clinical examination absence of both permanent mandibular canines and temporary biscuspid persistence. Lower left, accompanied by a slight dental malposition. In the radiographic exploration (panoramic), both right and left lower canines were observed, in a horizontal position, under the lower incisor apexes. We proceeded to the surgical extraction of both canines, passing the postoperative period without incidents. Satisfactory periodic and radiographic controls were performed at six months.
\end{abstract}

Keywords: Tooth, impacted; Tooth migration; Tooth, unerupted; Cuspid (source: MeSH NLM).

(c) Los autores. Este artículo es publicado por la revista Odontología Sanmarquina de la Facultad de Odontología, Universidad Nacional Mayor de San Marcos. Este es un artículo de acceso abierto, distribuido bajo los términos de la licencia Creative Commons Atribucion - No Comercia_Compartir Igual 4.0 Internacional. (http://creativecommons.org/licenses/by-nc-sa/4.0/) que permite el uso no comercial, distribución y reproducción en cualquier medio, siempre que la obra original sea debidamente citada. 


\section{Introducción}

Los caninos permanentes juegan un papel muy importante desde el punto de vista estético y funcional cuando están presentes en su posición normal. El fracaso de erupción del canino mandibular es un evento poco común, que ocurre aproximadamente 20 veces menos con respecto al canino maxilar ${ }^{1}$.

En general, la incidencia estimada del canino mandibular incluido es del 0,35 al 0,44\% y aún menos común es la migración del canino mandibular desde su posición normal al lado contrario cruzando la línea media; fenómeno conocido como transmigración ${ }^{2}$.

En cuanto a la frecuencia de aparición bajo los parámetros de sexo y edad, se observa que el sexo femenino es el más comúnmente afectado en una relación de 2:1, diagnosticándose con más frecuencia en pacientes menores de 20 años. Aparece mayoritariamente de forma unilateral, siendo los más afectados los caninos mandibulares izquierdos.

Mupparapu ${ }^{3}$ clasificó la transmigración de los caninos mandibulares en cinco tipos:

Tipo 1: Canino incluido mesioangularmente a través de la línea media, labial, o lingual a los dientes anteriores con la porción de la corona del diente que cruza la línea media.

Tipo 2: Canino incluido horizontalmente cerca del borde inferior de la mandíbula por debajo de los ápices de los incisivos.

Tipo 3: Caninos en erupción mesial o distal al canino opuesto.

Tipo 4: Canino incluido horizontalmente cerca del borde inferior de la mandíbula por debajo de los ápices del premolar o molar en el lado opuesto.
Tipo 5: Canino posicionado verticalmente en el centro con el eje largo del diente cruzando la línea media ${ }^{3-5}$.

La incidencia de estos tipos varía: el tipo $1(45,6 \%)$ siendo el más común, seguido por el tipo $2(20 \%)$, el tipo 4 $(17 \%)$, el tipo $3(14 \%)$ y el tipo $5(1,5 \%)^{5-8}$ (Figura 1 ).

A pesar de que los caninos transmigrados son generalmente asintomáticos, éstos pueden provocar el fenómeno de rizólisis por presión de las raíces de los dientes vecinos, la inclinación de dientes adyacentes, el síntoma neurálgico y el compromiso de estructuras que pueden causar dolor ${ }^{4}$.

Ayudar a esclarecer la etiología de esta entidad, el diagnóstico y tratamiento adecuado justifica la notificación de nuevos casos encontrados.

El objetivo de este trabajo es presentar un caso clínico de transmigración de caninos inferiores bilaterales del tipo 2 de la clasificación de Mupparapu que por su escasa frecuencia de aparición lo convierte en un fenómeno raro y de interés.

\section{Reporte del caso}

Acudió al servicio de Cirugía Bucal e Implantología en el 2016 un paciente varón de 16 ańos de edad remitido por su ortodoncista para valorar el tratamiento quirúrgico de ambos caninos mandibulares incluidos observados en exploración panorámica.

El paciente se encontraba asintomático, sin antecedentes patológicos personales ni familiares de interés que contraindicaran el tratamiento quirúrgico, niega antecedentes de traumas previos en la zona y refirió haber estado bajo observación desde el año 2011 por otros profesionales los cuales optaron por la abstención terapéutica.

A la exploración extraoral no se encontró ninguna alteración, e intraoralmente se observó malposición denta-

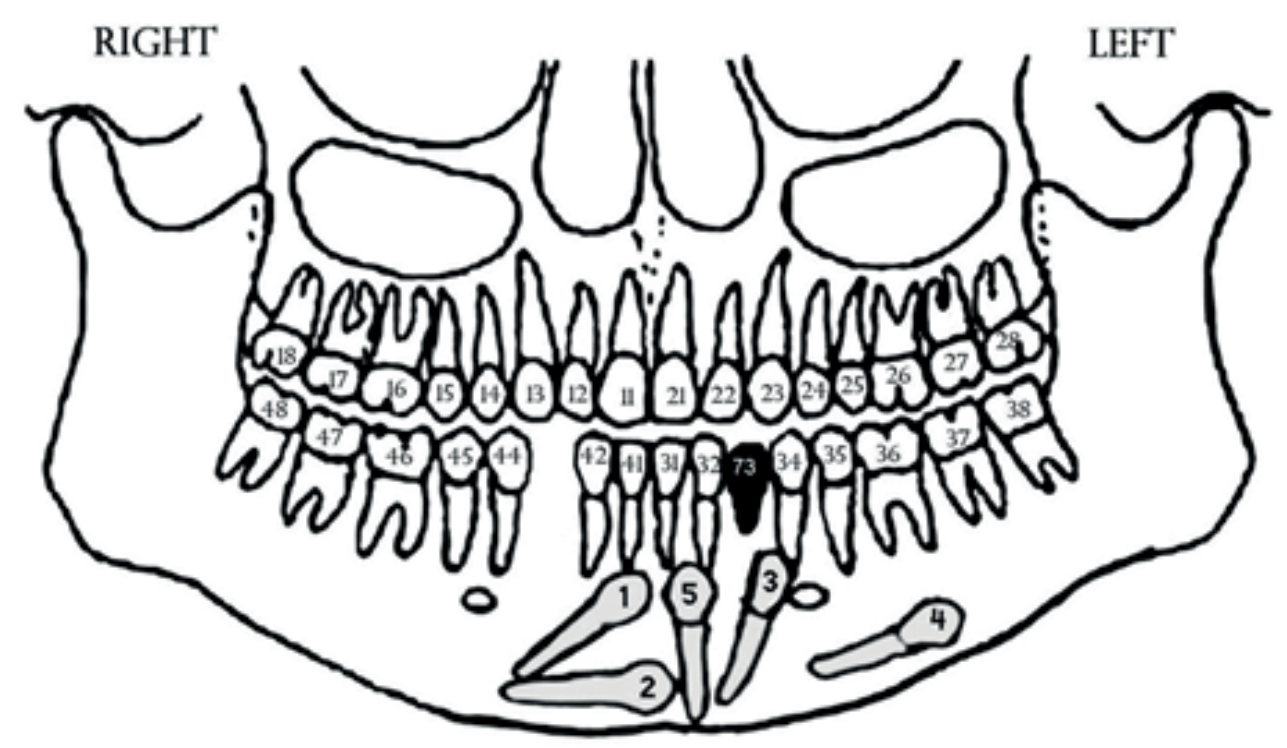

Figura 1. Representación esquemática de los cinco patrones distintos de transmigración (tipos 1-5). También se observa la presencia del diente temporal 73 y ausencia del 83 correspondiente con el caso clínico 
ria anteroinferior, ausencia de los caninos mandibulares derecho e izquierdo y persistencia del canino temporal izquierdo. No se evidenció ningún signo de movilidad dentaria o bolsas periodontales.

El paciente remitido por su ortodoncista aportó radiografías panorámicas previas desde el año 2011 hasta el 2016 (Figura 2 A, B, C y D), período en el que se había optado por la conducta expectante. En la radiografía más reciente (Figura 2 D) se observó la presencia de los dos caninos mandibulares en posición horizontal, situados bajo los ápices de incisivos inferiores, los cuales se correspondían con un patrón 2 de Mupparapu, con for- mación completa de las raíces, sin dilaceración ni reabsorción radicular de dientes adyacentes. Cabe destacar la retención dentaria de los cuatro terceros molares.

Se solicitó CBCT (Tomografía computarizada de haz cónico) para delimitar la posición exacta de los dientes, su estrecha relación con estructuras anatómicas adyacentes y llevar a cabo una correcta planificación quirúrgica (Figura 3).

Una vez obtenidas las pruebas diagnósticas, se informó al paciente y a sus padres de la necesidad de la extracción quirúrgica de los caninos, ante la imposibilidad
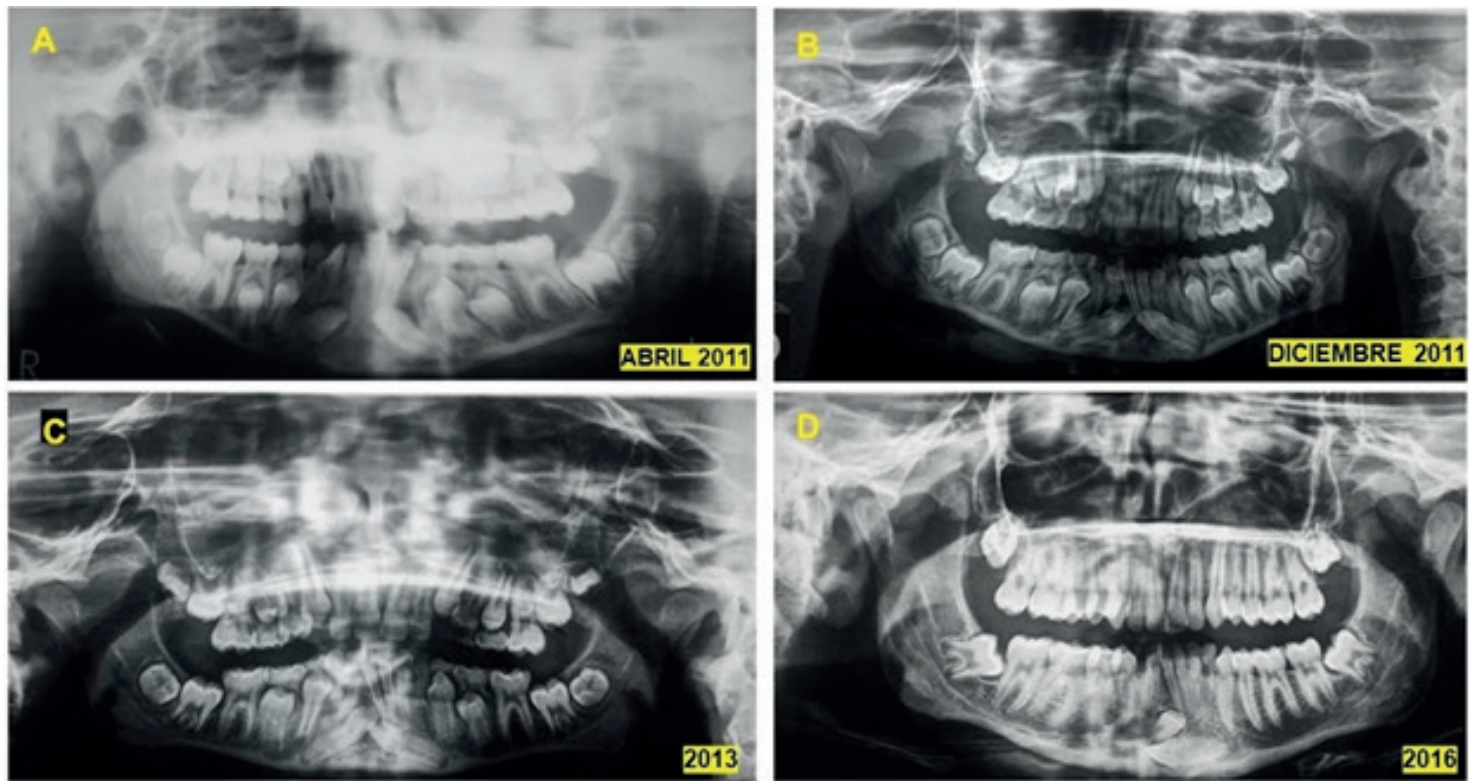

Figura 2. Radiografías panorámicas previas aportadas por el ortodoncista desde (2011-2016). A. Panorámica abril 2011, conducta expectante. B. Panorámica diciembre 2011, conducta expectante. C. Panorámica 2013, conducta expectante. Se observa un avance de la transmigración provocando desplazamiento de las raíces de incisivos inferiores. D. Panorámica 2016. Caninos transmigrados con un patrón 2 de Mupparapu
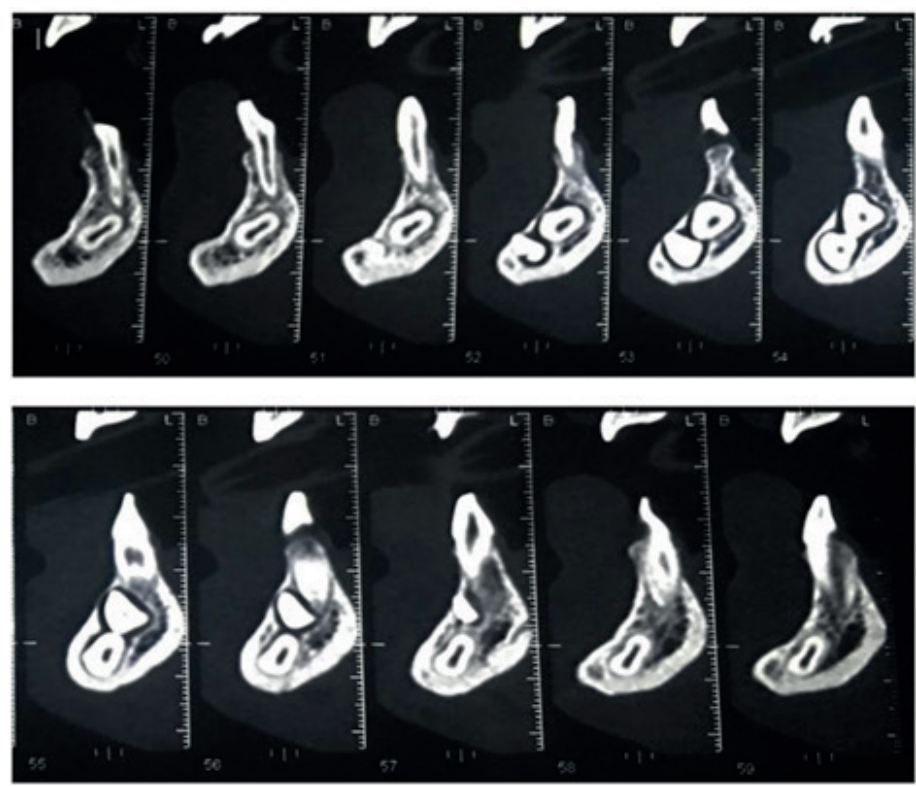

Figura 3. СВCT (Tomografía computarizada de haz cónico). Cortes Tomográficos. Se detalla la localización más vestibulizada del 43 con respecto al 33 
de realizar otro tratamiento conservador, se obtuvo el consentimiento informado por parte de sus padres en cumplimiento de la Ley 41/2002, básica reguladora de la autonomía del paciente y de derechos y obligaciones en materia de información y documentación clínica, debido a la minoría de edad del paciente, así como la autorización para la obtención de fotos con fines docentes. La historia clínica del paciente cumple con los requisitos según la Ley Orgánica 15/1999, de Protección de Datos.

El principal objetivo es la extracción quirúrgica de ambos caninos inferiores incluidos evitando así futuras complicaciones y permitiendo un posterior tratamiento ortodóntico satisfactorio.

Se pautó profilaxis antibiótica de amoxicilina $2 \mathrm{~g}$ una hora antes de la intervención quirúrgica.

Con signos vitales en parámetros normales y previa desinfección intraoral con enjuague de clorhexidina al $0,12 \%$ durante 1 minuto, se realizó una técnica anestésica mediante el bloqueo del nervio alveolar inferior de ambos lados, con articaína al $4 \%$ con epinefrina 1/100000. Se realizó incisión de Newman con despegamiento a espesor total, ostectomía vestibular con pieza de mano a baja velocidad utilizando fresa redonda de carburo de tungsteno y abundante irrigación, exposición de la corona del canino mandibular derecho, odontosección con fresa de fisura de tungsteno a nivel coronal facilitando la extracción, tracción del fragmento medio radicular con ayuda de una pequeña muesca y luxación mediante Winter y exéresis del tercio radicular. Ante la exposición del canino mandibular izquierdo localizado en posición lingual respecto al derecho, se procedió a su odontosección corono-cervical, luxación y extracción del fragmento coronal y finalmente la extracción del fragmento radicular. Se lavó el lecho quirúrgico con abundante suero fisiológico y se suturó con seda $4 / 0$, colocando un apósito compresivo en la zona del mentón para evitar la formación de hematomas (Figura 4).

La realización de múltiples odontosecciones tuvo como objetivo ocasionar el menor defecto óseo posible y la eliminación completa de ambos caninos transmigrados. La intervención se realizó sin complicaciones con una duración de aproximadamente 45 minutos.

Se entregaron al paciente las indicaciones postoperatorias y prescripción de analgesia de rescate (paracetamol de $650 \mathrm{mg}$ ) y antiinflamatorios (ibuprofeno $400 \mathrm{mg}$
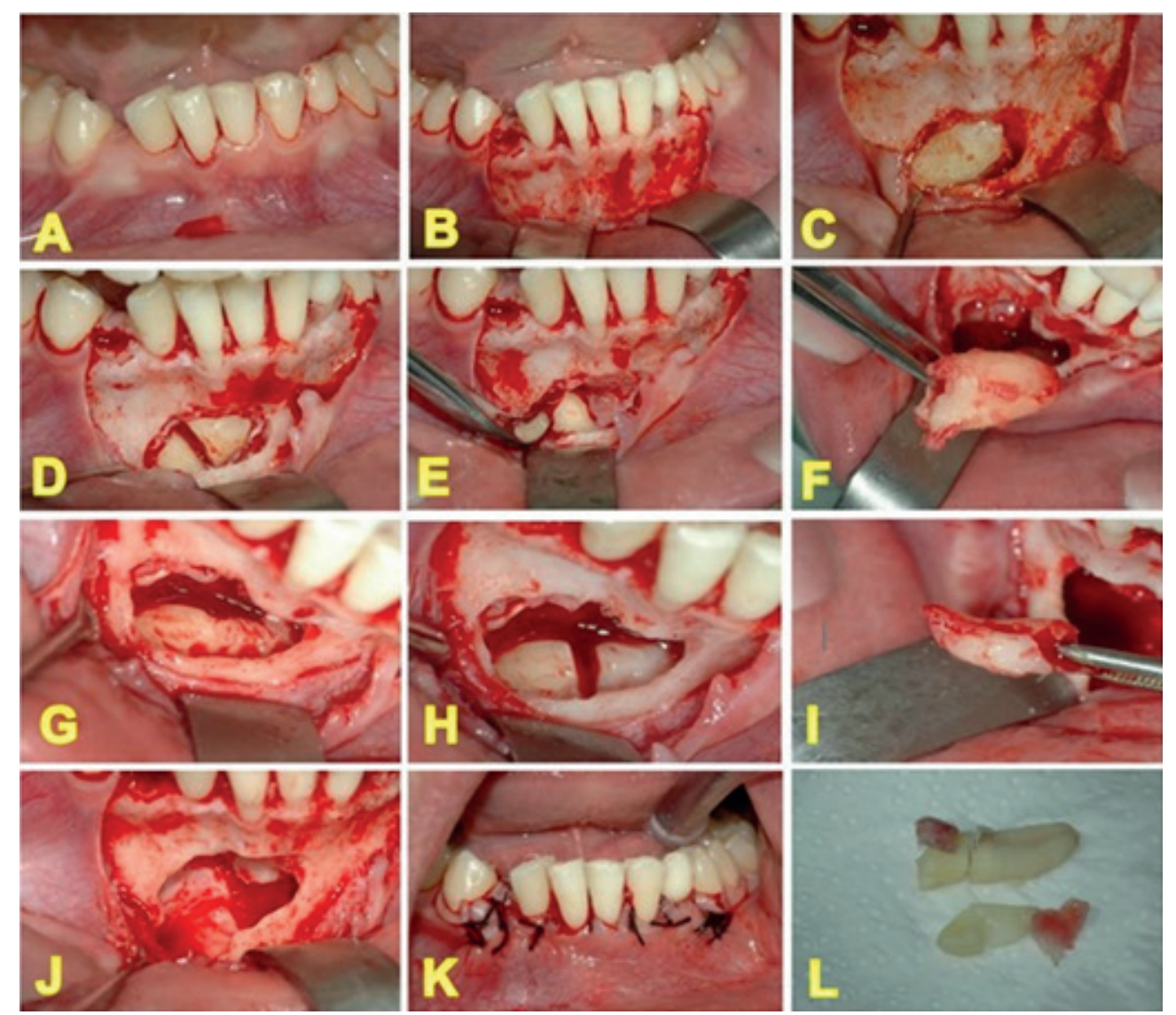

Figura 4. A. Imagen intraoral. Ausencia de ambos caninos permanentes, persistencia del 73, e inclinación y apiñamiento de incisivos antero-inferiores. B. Incisión Newman Completa y Despegamiento Mucoperióstico. C. Ostectomía y exposición de la corona del canino mandibular derecho. D. Odontosección a nivel coronal facilitando la extracción con mínima ostectomía. E. Tracción del fragmento medio radicular, con ayuda de una pequeña muesca y luxación mediante Winter. F. Excéresis del tercio radicular. G. Exposición del canino mandibular izquierdo 33 localizado en posición lingual respecto al derecho. H. Odontosección corono-cervical del 33. I. Extracción del fragmento radicular 33. J. Limpieza con suero fisiológico y legrado de la cavidad. K. Sutura de la herida con puntos simples y seda 4/0. L. Reconstrucción de los fragmentos dentarios y correspondientes sacos pericoronarios 
cada 8 horas durante 3-4 días), las primeras 24 horas se aconsejó evitar el uso de enjuagues y a partir de las 24 horas, se indicó higiene con cepillo quirúrgico en la zona de la herida y colutorios de clorhexidina al $0,12 \%$.

Se retiró la sutura a los 7 días realizándose controles clínicos sucesivos a los 15 días, al mes, a los 3 meses y radiográficos a los 6 meses y 1 año para controlar la regeneración ósea de la zona (Figuras 5 y 6 ).

\section{Discusión}

Según Revelo y Espinosa "la etiología y mecanismo exacto de la transmigración aún no está claro, aunque se han propuesto un cierto número de teorías, en general, se acepta que el diente incluido sigue la trayectoria de menos resistencia. El diente se mueve en la dirección de la corona y la inclinación mesial del folículo hace posible que el diente adopte una posición horizontal, migrando hacia el lado contrario".
Otros factores tales como la forma cónica del canino, ausencia congénita de incisivos laterales, lesiones quísticas o tumorales relacionados con el canino, pérdida prematura de los caninos temporales o conservación anormal de éstos, discrepancias de la longitud del arco respecto al tamaño del diente, y otras condiciones patológicas como tumores, odontomas, quistes y un pequeño obstáculo, tal como un fragmento de la raíz, serían suficientes para desviar un diente de su trayectoria normal de la erupción ${ }^{5}$.

La asociación entre la transmigración bilateral de caninos y la presencia de un quiste dentígero es posible aunque poco frecuente, y teniendo en cuenta que éstos pueden llegar a ser extensos causando abombamiento, erosión de corticales óseas y rizólisis de dientes adyacentes, el diagnóstico y tratamiento precoz puede evitar futuras complicaciones ${ }^{9,10}$. En este caso no se evidenció en ninguna exploración radiográfica la presencia de ri-

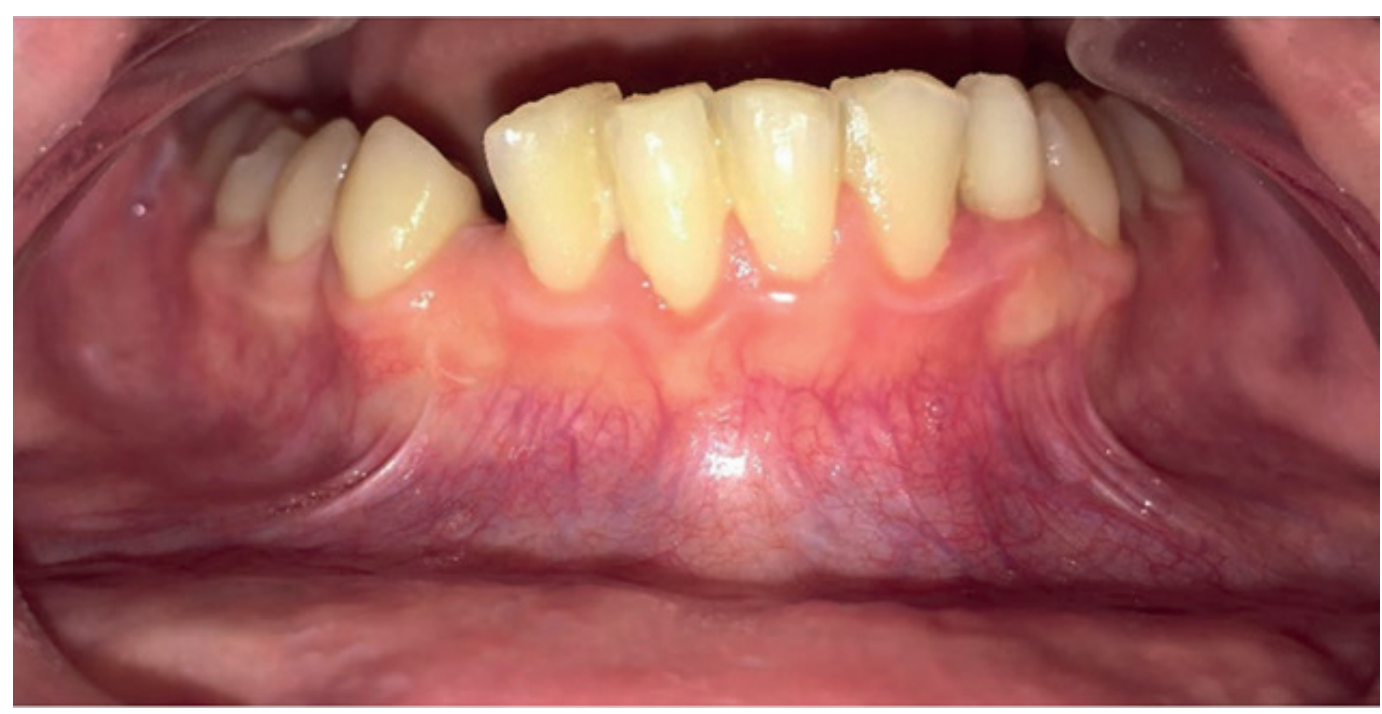

Figura 5. Panorámica de control 6 meses después. Se observa una adecuada regeneración ósea de la zona intervenida

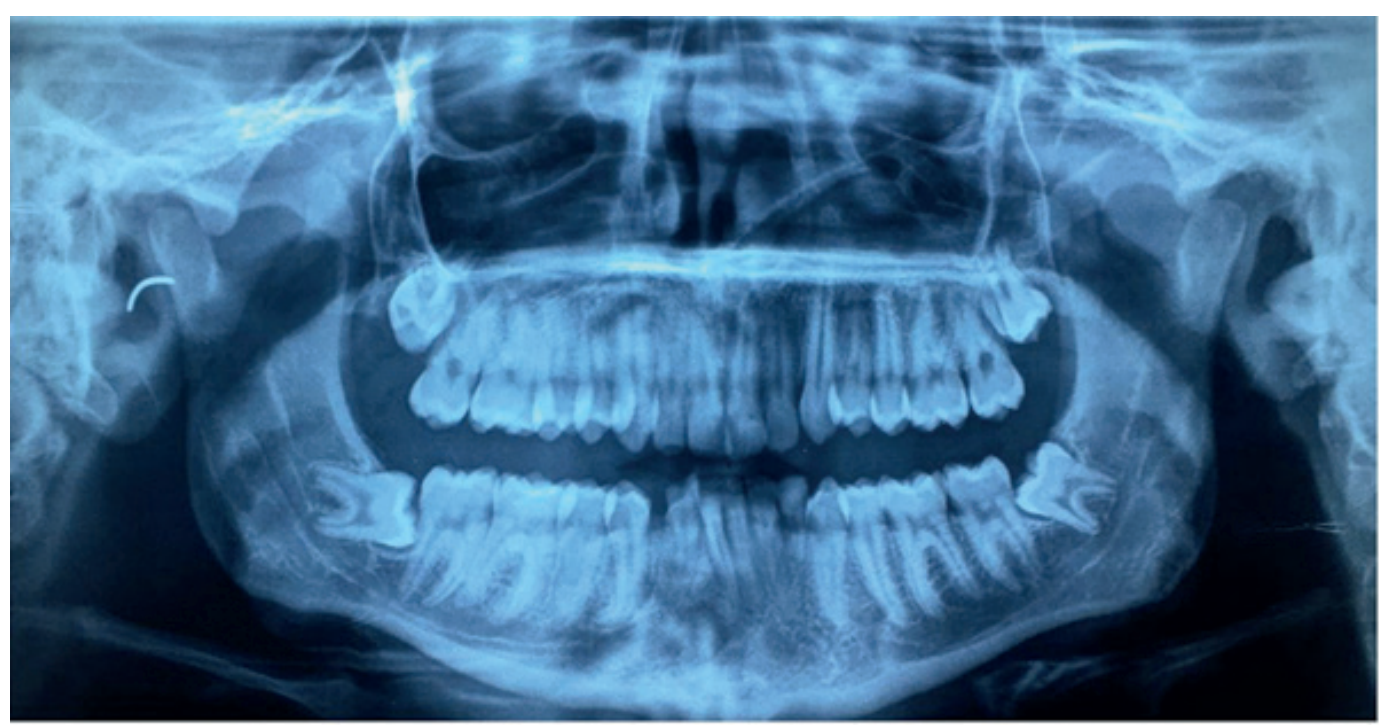

Figura 6. Imagen intraoral durante la revisión del paciente 6 meses después donde se observa una adecuada cicatrización de los tejidos blandos 
zólisis de dientes adyacentes ni asociación con quistes dentígeros que pudieran causar expansión o erosión de corticales óseas.

La transmigración también puede aparecer relacionada con odontomas. La aparición de odontomas no diagnosticados ni tratados durante el desarrollo y erupción de los caninos puede dar lugar a su inclusión y posterior transmigración.

Madiraju y Singamaneni ${ }^{11}$ en 2013 y Salamanca et al. ${ }^{12}$ en 2017 publicaron casos donde la presencia de odontomas relacionados con el canino temporal parece haber sido la causa de la transmigración canina. En el presente caso tampoco se encontró la asociación entre ambas entidades.

Los otros factores clínicos asociados con la transmigración canina son agenesia de los incisivos laterales, inclinación de incisivos y línea media dental desviada. Cuando dos o más de estas características están presentes y el paciente tiene entre 10 y 13 ańos, debe ser evaluado radiográficamente por la sospecha de inclusión y transmigración del canino ${ }^{13}$. Las exploraciones radiográficas se iniciaron en este caso debido a la presencia de estos signos.

De manera tradicional se han venido utilizando distintas técnicas radiográficas para el diagnóstico de inclusiones dentarias, entre ellas la radiografía retroalveolar o periapical que resultaría insuficiente en el caso de los caninos transmigrados muy alejados de la zona habitual de erupción al igual que la radiografía oclusal y panorámica que se han visto desplazadas por nuevas técnicas computarizadas.

En la actualidad las técnicas tomográficas como la CBCT es la opción más adecuada para determinar el estado de los dientes vecinos, proporcionar imágenes tridimensionales de alta calidad, a tamaño real, sin superposiciones y con una relación coste/beneficio satisfactoria ${ }^{13-14}$.

En este caso se ha seguido el protocolo de diagnóstico a través de radiografías panorámicas previas aportadas por el ortodoncista, complementando el diagnóstico definitivo con la CBCT que permitió evaluar el estado de la inclusión, pronóstico de la misma y la actitud terapéutica a seguir.

En relación al tratamiento del canino mandibular transmigrado depende de numerosos factores, tales como: edad de diagnóstico, condiciones socio-económicas, patologías asociadas, profundidad de la inclusión etc., por lo que se pueden establecer las siguientes opciones:

Observación periódica: terapia a seguir en niños con dentición mixta o temporal (7-10 años) con caninos desplazados y en posición intraósea anómala. En este caso se debe esperar su tiempo de erupción definitivo. Ante la sospecha de antecedentes familiares de retenciones o falta de espacio debe solicitarse un control radiográfico ${ }^{14}$.

Tratamiento preventivo e interceptivo: se indica en fase de dentición mixta tardía (10-13 años), consistien- do en extracciones seriadas, mantenedores de espacio, etc., favoreciendo así la erupción fisiológica ${ }^{14}$.

Exposición quirúrgica y tratamiento de ortodoncia: cuando no se han obtenido los resultados esperados con los tratamientos anteriores, el diagnóstico se realiza de forma más tardía, o los problemas de erupción se deben a obstáculos mucosos, dentarios u otras patologías, la eliminación de dichos obstáculos puede permitir que el canino erupcione en una posición adecuada en la arcada ${ }^{14}$.

En ausencia de circunstancias anteriores la tracción quirúrgica ortodóncica adquiere un papel relevante y requiere una valoración interdisciplinar (ortodoncista, cirujano, y periodoncista).

Autotrasplante: si los incisivos mandibulares están en posición normal y el espacio para el canino transmigrado es suficiente. Actualmente es una opción terapéutica prácticamente inexistente debido al alto índice de fracaso y al alto porcentaje de éxito de los implantes dentales 15 .

Extracción quirúrgica: una vez que la corona del canino migra más allá del incisivo contralateral o el ápice del canino se encuentra más allá del ápice del incisivo lateral adyacente, ortodóncicamente es imposible reposicionar el diente, o en los casos en que el canino transmigrado está asociado con patología o síntomas neurológicos ${ }^{15}$.

Abstención terapéutica: conducta terapéutica con una gran controversia por la que se inclinan numerosos profesionales ya sea por desconocimiento de las técnicas quirúrgicas o por considerar que es la mejor opción en ausencia de manifestaciones clínicas o radiológicas. "La presencia de caninos superiores incluidos en ausencia de manifestaciones, deberían ser controlados en el tiempo. Situación diferente para los caninos incluidos mandibulares" ${ }^{14}$.

Una revisión sistemática publicada en 2016 por Dalessandri et al. fue contundente a la hora de determinar que, "la abstención terapéutica no debe ser contemplada para los caninos mandibulares incluidos y por consiguiente, deben ser extraídos. Sin embargo, cuando éstos hayan sufrido el "fenómeno de transmigración" esta actitud expectante y de seguimiento sería válida" ${ }^{16}$.

Dada la importancia del canino, su no tratamiento podría conllevar un compromiso estético, fonético y masticatorio. Otras complicaciones de no tratar los caninos incluidos pueden concluir en un desplazamiento o pérdida de vitalidad de los incisivos, acortamiento del arco dental, formación de quistes foliculares, anquilosis del canino, infecciones y dolores recurrentes, reabsorción interna, reabsorción externa del diente adyacente o combinaciones de todos estos factores. Por otra parte, la presencia de caninos incluidos puede no causar efectos perjudiciales durante toda la vida del paciente. No obstante el potencial riesgo de complicaciones recalca la necesidad de tratamiento o monitorización de éstos ${ }^{17}$.

Basados en este caso y teniendo en cuenta la evolución de la patología desde el diagnóstico inicial, se puede establecer como protocolo recomendable, que ante una 
detección precoz de la inclusión, el tratamiento interceptivo será la opción más adecuada con revisiones clínico - radiológicas que permitan actuar con rapidez. De esta forma las opciones de tratamiento aumentan en gran medida, siendo la cirugía de rescate o el tratamiento quirúrgico ortodóncico el más adecuado, opción que tal vez pudo ser valorada cuando el paciente acudió por primera vez a otro servicio en el cual se le aconsejó la abstención terapéutica.

Cuando aún no se ha establecido la transmigración, pero se sospecha que ésta puede tener lugar, una valoración interdisciplinar y el esfuerzo por devolver los caninos a su posición fisiológica seria el tratamiento de elección.

Una vez establecida la transmigración existen controversias entre la extracción quirúrgica para evitar futuras complicaciones o la observación periódica como se ha hecho referencia anteriormente.

En este caso se decidió la extracción de ambos caninos a pesar de la ausencia de sintomatología, ya que la inclusión había provocado malposición dentaria anteroinferior que el paciente quería tratar y una vez explicadas las posibles complicaciones relacionadas con la inclusión y las relacionadas con la cirugía, el paciente decidió realizarse el tratamiento quirúrgico.

Es oportuno destacar que debido a la complejidad de la cirugía y a las posibles complicaciones derivadas de ella, tanto de tejidos blandos, dentarios, óseos, vasculares y nerviosos, el tratamiento quirúrgico debe ser minuciosamente planificado y llevado a cabo por profesionales con experiencia para obtener resultados predecibles.

Se concluye que la doble transmigración de caninos mandibulares es un evento poco común y generalmente asintomático.

Ante la sospecha clínica de esta anomalía, la exploración radiográfica para establecer un diagnostico precoz es de vital importancia para poder ser tratada y evitar futuras complicaciones.

\section{Referencias bibliográficas}

1. Qaradaghi IF. Bilateral transmigration of impacted mandibular canines: Report of two cases and review. Rev Clín Pesq Odontol. 2010;6(3):271-75.

2. Revelo Pérez LA, Espinosa Morales JG. Transmigration of the mandibular canine. Report of a clinical case and review of the literature. Rev ADM. 2013;70(4).

3. Mupparapu M. Patterns of intra-osseous transmigration and ectopic eruption of mandibular canines: review of literature and report of nine additional cases. Dentomaxillofac Radiol. 2002;31:355-60. doi:10.1038/sj.dmfr.4600732
4. Jayanthi P, Shamsudeen SSM, Rooban T, Umadevi M, Ranganathan K. Transmigration of mandibular canines: A case report. Indian J Multidiscip Dent. 2011;1(4):219-21.

5. Bhullar MK, Aggarwal I, Verma R, Uppal AS. Mandibular canine transmigration: Report of three cases and literature review. J Int Soc Prevent Communit Dent. 2017;7(1):8-14.

6. Kumar S, Jayaswal P, Pentapati KC, Valiathan A, Kotak $\mathrm{N}$. Investigation of the transmigrated canine in an orthodontic patient population. J Orthod. 2012;39(2):89-94.

7. Isa-Kara M, Ay S, Murat-Aktan A, Sener I, Bereket C, Ezirganli S, Demirkol M. Analysis of different type of transmigrant mandibular teeth. Med Oral Patol Oral Cir Bucal. 2011; May 1;16 (3):e335-40. doi:10.4317/medoral.16.e335

8. Vuchkova J, Farah CS. Canine transmigration: comprehensive literature review and report of 4 new Australian cases. Oral Surg Oral Med Oral Pathol Oral Radiol Endod. 2010;109:e46-e53.

9. Omami G, Mathew R. Kissing Canines Associated with a Dentigerous Cyst: Case Report and Mini Review. Int J Dent Oral Sci. 2015;2(6):84-6.

10. Shetty UA, Bhandary S, D'Cruz AM. Dentigerous Cyst Associated with Bilaterally Impacted Permanent Mandibular Canines: A Report of a Rare Case. Int J Clin Prev Dent. 2014;10(4):281-4.

11. Madiraju GS, Rao KS, Singamaneni V. A rare case of transmigration of mandibular canine associated with an odontoma. BMJ Case Rep. 2013. doi: 10.1136/bcr2013-009658.

12. Salamanca C, Ottone NE, Arias Rubilar C, Garay I, Fuentes R. Caninos Transmigrados Asociados a Odontomas. Int J Morphol. 2017;35(2):465-8. Doi:10.4067/ S0717-95022017000200013.

13. Umashree N, Kumar A, Nagaraj T. Transmigration of mandibular canines. Case Rep Dent. 2013;2013:697671. doi: 10.1155/2013/697671.

14. Barona Dorado C, Buesa Bárez JM, Fernández Cáliz F, Martínez-González JM. Opciones terapéuticas. En: Normon, S.A, editores. Dientes retenidos II (caninos). $1^{\text {a }} \mathrm{ed}$. Madrid: Laboratorios Normon, S.A; 2018. p. 121-9

15. Elhag SB, Abdulghani AS. Primary failure of eruption combined with bilateral transmigration of mandibular canines, transposition, torus palatinus, and class III incisor relationship: A rare case report. Eur J Dent. 2015 Oct-Dec;9(4):594-8. doi: 10.4103/1305-7456.172628.

16. Dalessandri D, Parrini S, Rubiano R, Gallone D, Migliorati $M$. Impacted and transmigrant mandibular canines incidence, aetiology, and treatment: A systematic review. Eur J Orthod. 2017 Apr 1; 39(2):161-9

17. Egido Moreno S, Arnau Matas C, Juárez Escalona I, Jané-Salas E, Marí Roig A, López-López J. Caninos incluidos, tratamiento odontológico. Revisión de la literatura. Av. Odontoestomatol. 2013;29(5):227-238. 
